\title{
Evaluation of GPS/BDS indoor positioning performance and enhancement
}

\author{
He, Zhe; Petovello, Mark; Pei, Ling; Olesen, Daniel Haugård
}

Published in:

Advances in Space Research

Link to article, DOI:

10.1016/j.asr.2016.09.009

Publication date:

2017

Document Version

Peer reviewed version

Link back to DTU Orbit

Citation (APA):

He, Z., Petovello, M., Pei, L., \& Olesen, D. H. (2017). Evaluation of GPS/BDS indoor positioning performance and enhancement. Advances in Space Research, 59(3), 870-876. https://doi.org/10.1016/j.asr.2016.09.009

\section{General rights}

Copyright and moral rights for the publications made accessible in the public portal are retained by the authors and/or other copyright owners and it is a condition of accessing publications that users recognise and abide by the legal requirements associated with these rights.

- Users may download and print one copy of any publication from the public portal for the purpose of private study or research.

- You may not further distribute the material or use it for any profit-making activity or commercial gain

- You may freely distribute the URL identifying the publication in the public portal

If you believe that this document breaches copyright please contact us providing details, and we will remove access to the work immediately and investigate your claim 


\section{Accepted Manuscript}

Evaluation of GPS/BDS Indoor Positioning Performance and Enhancement

Zhe He, Mark Petovello, Ling Pei, Daniel M. Olesen

PII:

S0273-1177(16)30522-1

DOI:

http://dx.doi.org/10.1016/j.asr.2016.09.009

Reference:

JASR 12905

To appear in:

Advances in Space Research

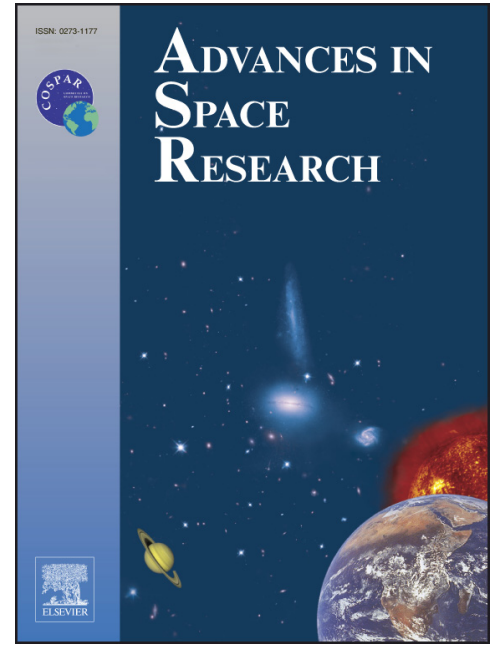

Received Date:

18 February 2016

Revised Date:

8 September 2016

Accepted Date:

10 September 2016

Please cite this article as: He, Z., Petovello, M., Pei, L., Olesen, D.M., Evaluation of GPS/BDS Indoor Positioning Performance and Enhancement, Advances in Space Research (2016), doi: http://dx.doi.org/10.1016/j.asr. 2016.09.009

This is a PDF file of an unedited manuscript that has been accepted for publication. As a service to our customers we are providing this early version of the manuscript. The manuscript will undergo copyediting, typesetting, and review of the resulting proof before it is published in its final form. Please note that during the production process errors may be discovered which could affect the content, and all legal disclaimers that apply to the journal pertain. 


\title{
Evaluation of GPS/BDS Indoor Positioning Performance and Enhancement ${ }^{\text {tw }}$
}

\author{
Zhe $\mathrm{He}^{1, *}$
}

PLAN group, Geomatics Engineering, University of Calgary

Mark Petovello ${ }^{2, *}$

PLAN group, Geomatics Engineering, University of Calgary

$$
\text { Ling Pei }{ }^{3}
$$

Shanghai Key Laboratory of Navigation and Location-based Services, School of Electronic Information and Electrical Engineering, Shanghai Jiao Tong University

Daniel M. Olesen

DTU space, Technical University of Denmark

\begin{abstract}
This paper assesses the potential of using BDS and GPS signals to position in challenged environments such as indoors. Traditional assisted GNSS approaches that use code phase as measurements (i.e., coarse-time solutions) are shown to be prone to multipath and noise. An enhanced approach that has superior sensitivity and positioning performance - the so-called direct positioning receiver architecture - has been implemented and evaluated using live indoor BDS and/or GPS signals. Real indoor experiments have been
\end{abstract}

Revised paper for journal Advances in Space Research.

* Corresponding author

Email addresses: zhehe@ucalgary.ca (Zhe He ), mark.petovello@ucalgary.ca

(Mark Petovello), ling.pei@sjtu.edu.cn (Ling Pei), danole@space.dtu.dk (Daniel M. Olesen)

${ }^{1}$ PLAN Group, Geomatics Engineering Department, 2500 University Dr. NW, Calgary, Alberta, Canada, T2N 1N4

${ }^{2}$ PLAN Group, Geomatics Engineering Department, 2500 University Dr. NW, Calgary, Alberta, Canada, T2N 1N4

${ }^{3}$ WeiDianZhi Building 309G, Shanghai Jiao Tong University, Shanghai, China, 200240 
conducted in Shanghai and significant improvement has been observed with enhanced approaches: results with BDS constellation show better horizontal positioning performance (biases are less than 10 metres) than using GPS alone, but are slightly worse in the vertical axis; when using the enhanced approach with BDS and GPS, both horizontal and vertical axes show promising results for the environments considered herein; the coarse-time state converges faster and is more reliable compared to other solutions.

Keywords: assisted GNSS; indoor positioning; indoor BDS

\section{Introduction}

In the past, the concept of assisted-GPS significantly broadened the applications for mass market GPS receivers. Typically, the signal reception sensitivity is enhanced for each individual satellite channel with long noncoherent integration Van Diggelen (2009). Few results have been found (to the best of authors' knowledge) in the literature evaluating or comparing the positioning performance using assisted BeiDou (BDS), assisted GPS or both, in real indoor environments. This could be due to the fact that BDS MEO satellites are not fully established globally. However, in eastern China, the coverage of BDS is good enough to evaluate such performance. In the past, the benefits of five GEO satellites in China area for positioning indoors have not been mentioned or discussed either. Moreover, current literature lacks discussing of the methods to further enhance the receiver's performance using single or multiple constellations based on live indoor data.

To improve signal tracking performance, standard approaches include increase integration, such as using high quality oscillator and increasing long coherent integration are reported in Pany et al. (2009), and Gaggero \& Borio (2008); or use advanced receiver architectures such as vector tracking, ultratight integration with sensors as discussed in Lashley et al. (2009) and Pany \& Eissfeller (2006) to name a few. In the meantime, some researchers also suggest to use all-in-view satellite to enhance the acquisition performance in GNSS receivers known as 'collective detection' as reported in Axelrad et al. (2011), Cheong et al. (2012), and P. Esteves \& Ries (2014). One interesting by-product for this direct approach is that the positioning or navigation solution can also be determined during the acquisition or detection process. For these collective detection oriented algorithms, a chip spacing of 0.5 are often used which gives the worst case and average correlation losses about $2.5 \mathrm{~dB}$ 
and $1.16 \mathrm{~dB}$ respectively as shown in De Wilde et al. (2006). To the contrary, others have focused on improving estimation performance of this direct approach as a maximum likelihood positioning method (evaluate the likelihood function in some region) or maximum a posterior method (making use of a prior information recursively), such as reported in Closas et al. (2007), He \& Petovello (2014) and He (2013). Multiple correlator is often used by evaluating multiple correlator output within interested region van Graas et al. (2005). The multiple correlator approach for code phase estimation turns out to be a straight forward ML code phase estimator. Analogously, the direct positioning approach also can be used for the Doppler and velocity determination with multiple constellations, and the corresponding results in different indoor environments have been discussed in He et al. (2012). By using above-mentioned approaches, weak GPS signals can be more reliably tracked, which also have other potentials such as reflectometry reported in Jin \& Najibi (2014) or indoor navigation He et al. (2013), Zhuang et al. (2015).

The first objective of paper is to evaluate the performance of assisted GPS/BDS (A-GPS/BDS) traditional architecture in real harsh environments. Blockage, fading or multipath can all occur simultaneously resulting in signals having a carrier to noise-density ratio $\left(\mathrm{C} / \mathrm{N}_{0}\right)$ lower than $15 \mathrm{~dB}-\mathrm{Hz}$. The paper also implements a direct positioning receiver architecture similar to that in He (2013); He \& Petovello (2014) which is an enhanced assisted receiver. The performance of enhanced A-GPS, A-BDS and A-GPS/BDS are also assessed and compared to the traditional approach in real harsh environments.

Section 2 briefly discusses the traditional assisted GPS/BDS technology and also outlines the enhanced approach. Section 3 shows the experiments conducted in Shanghai and illustrates indoor environments used. In Section 4, data processing results are presented and the corresponding positioning statistics are summarized for comparison. Finally, Section 5 concludes the paper.

\section{Traditional and Enhanced Assisted-GNSS}

The traditional A-GPS technology has been discussed in Van Diggelen (2009) and typically uses code phase as measurements instead of pseudorange. Code phase observations have millisecond ambiguities since accurate time of transmission information is not required when constructing the mea- 
surements. The A-GPS will use network information to get fine time assistance in sub-second level, and ephemeris or satellite positions/velocities. Using this information, the receiver only needs to evaluate the correlation between incoming signal and local replica within a much smaller region. Long non-coherent integration is commonly used to improve the reception sensitivity and quality of the observations.

It is also known that using standard delay-lock loops with strong signals will tend to yield a good approximation of maximum likelihood code phase estimators as reported in Parkinson (1996). For the indoor scenarios considered herein, multiple correlators are used to track the code phase errors over observation intervals whose duration, $T_{I}$, depends on both the coherent integration time $\left(T_{C I}\right)$ and number of noncoherent combinations, $P$. In Hurd et al. (1987), it has shown that the maximum likelihood code phase estimate, $\hat{\tau}_{M L}$, can be expressed as:

$$
\hat{\tau}_{M L}=\underset{\tau}{\operatorname{argmax}}\left|\int_{0}^{T_{C I}} \tilde{r}(t) c(t-\tau) d t\right|^{2}
$$

where $c(t)$ is the local code sequence, $\tau$ is the code phase and $\tilde{r}(t)$ is the complex envelope that is obtained by performing quadrature decomposition Proakis (2001). In this equation, the Doppler is assumed to be accurately compensated for in order to simplify the following analysis. The in-phase and quadrature correlator outputs for a coherent integration of $T_{C I}$ can be defined as:

$$
\begin{gathered}
y_{I, p}(\tau)=\operatorname{Re}\left(\int_{(p-1) T_{C I}}^{p T_{C I}} \tilde{r}(t) c(t-\tau) d t\right) \\
y_{Q, p}(\tau)=\operatorname{Im}\left(\int_{(p-1) T_{C I}}^{p T_{C I}} \tilde{r}(t) c(t-\tau) d t\right)
\end{gathered}
$$

With correlator outputs defined above, the correlator power at code phase $\tau$ for the $p^{\text {th }}$ coherent integration interval of $T_{C I}$ can be defined as

$$
z_{p}(\tau)=y_{I, p}^{2}(\tau)+y_{Q, p}^{2}(\tau)
$$

Then the maximum likelihood estimate of code phase for a total integration time of $T_{I}=P T_{C I}$ can be expressed as:

$$
\hat{\tau}_{M L}=\underset{\tau}{\operatorname{argmax}} \sum_{p=1}^{P} z_{p}(\tau)
$$


The standard single point positioning navigation solution takes pseudorange measurements as inputs, and thus only four states are estimated: three user position terms and the unknown clock bias between the satellite and receiver. If decoding of navigation message is not possible in weak signal environments such as indoors, a coarse-time navigation solution is usually used which requires estimating the coarse timing error. The state for standard coarse time state vector will be

$$
\delta \theta=\left[\begin{array}{lllll}
\delta E & \delta N & \delta U & \delta t_{b} & \delta t_{c}
\end{array}\right]^{T}
$$

where $\delta E, \delta N$ and $\delta U$ are the position errors in the local-level frame (east, north and vertical directions respectively); and $\delta t_{b}$ and $\delta t_{c}$ are, respectively, the clock bias and coarse-time states. The observation equation relates the code phase measurements to these states Van Diggelen (2009):

$$
\delta \tau_{k}=\tau_{k}-\hat{\tau}_{k}=H_{k} \delta \theta+w_{k}=\left[-\mathbf{u}_{\mathbf{k}}, 1, \dot{\rho}_{k}\right] \delta \theta+w_{k}
$$

In Equation (6), $\tau_{k}$ is the code phase observation for $k^{\text {th }}$ satellite, $\hat{\tau}_{k}$ is the predicted code phase for this satellite, $H_{k}$ is the design matrix for this observation, $\mathbf{u}_{k}$ is the unit vector from the satellite to the receiver and $\dot{\rho}_{k}$ is the range rate for this satellite. For the coarse-time solution, the millisecond ambiguity has to be solved; a detailed procedure to correctly resolve this millisecond ambiguity is explained in Van Diggelen (2009).

To further enhance the receiver's sensitivity, researchers have proposed using multiple satellites in-view and non-coherently combining them directly in the position domain. These methods are commonly referred to as collective detection from an acquisition perspective Axelrad et al. (2011), or maximum likelihood positioning from an estimation perspective Closas et al. (2007).

In the enhanced A-GPS/BDS architecture, multiple correlators are used to enhance the tracking sensitivity. Then blocks of correlators are also used as measurements to the navigation solution. By using Bayesian filter theory, a posterior likelihood ratios can be computed by using a prior information and incoming correlations as input. Then, maximum a posterior detection and estimation is conducted simultaneously in the solution determination stage in order to get the optimal solution.

In the direct coarse time positioning receiver, given some a priori state information $\theta_{0}$, all the correlator values for different satellites are used to estimate a coarse-time solution state $\delta \theta$ as shown in (5). Assuming there are 
$M$ satellites available for determination of the coarse-time solution, then the maximum likelihood estimate of the state vector can be written as:

$$
\hat{\theta}_{M L}=\underset{\theta}{\operatorname{argmax}} \sum_{m=1}^{M}\left(\sum_{p=1}^{P} z_{p}^{m}(\tau(\theta))\right)
$$

In (7), each code phase value $\tau$ for each individual satellite is directly related with a state $\theta$; the linearized relationship is already shown in (6). It can be seen that the direct coarse-time positioning approach is searching over all the possible solutions in some admissive region.

For example, in the beginning, we can specify a flat priori probability $\pi^{-}$ distribution over interested region $S$. The enhanced approach will need to evaluate the a posteriori probabilities $\pi$ for each candidate points within this region. The total region can be expressed as $S^{+}=S+\phi$ where $\phi$ is the null space, then we will have $\int_{S} \pi(s) d s+\pi(\phi)=1$. Typical maximum likelihood positioning approach will need to evaluate the position domain likelihood function $L(Y \mid s)$ for $s \in S^{+}$, where $Y$ is the correlator values. Our enhanced approach is based on maximum a posteriori probability (MAP), which means recursively a priori information is used for calculating a posteriori probability as well as solution estimate. Position domain a posteriori probability at $k$ epoch can be updated using $\pi(k, s)=\frac{\pi^{-}(k, s) L(Y \mid s)}{C}$ where $\mathrm{C}$ is the normalization term. To do this, the correlator values $Y$ corresponding to each searching point $s \in S^{+}$is required. After estimating the position solution, a Kalman filter can be used to propagate the solution over time in order to make best use of current and past information. A detailed procedure for the implementation can be found in He (2013); He \& Petovello (2014). Regarding the implementation parameters, the short coherent integration times are used, long non-coherent combining is then applied in order to achieve the predefined detection rate give certain false alarm rate. Typically, coherent integration time is set as $3 \mathrm{~ms}$ to $7 \mathrm{~ms}$, total observation time $T_{O}$ is $0.8 \mathrm{~s}$ to $1.2 \mathrm{~s}$. The code phase search spacing are between 0.015 to 0.05 chips considering different sensitivity and tracking stages.

\section{Indoor Environments and Data Collection}

A few indoor/outdoor experiments have been conducted in the MingHang campus of Shanghai Jiao Tong University. The primary pieces of equipment were a dual frequency front-end that can capture civilian GPS L1 and BDS 
B1 signals, an antenna and a reference receiver. The down-converted intermediate frequency (IF) samples are then processed by software receivers that implement different algorithm as discussed in Section 2.

Overall, results showed that in open sky environments the traditional approach has similar performance to the enhanced methods. In contrast, difficult environments such as when the receiver was surrounded by tall buildings showed that the traditional approach can be severely affected by multipath and is less robust/accurate than the enhanced approached. To emphasize improvements of the proposed architecture, only the results from the most challenging environments are shown and discussed in the following sections.

The indoor environment for data collection is shown in Figure 1. Typically, all satellites are experiencing blockage/multipath/fading. Most satellite signals are in the range of 15 to $35 \mathrm{~dB}-\mathrm{Hz}$ during the experiments which indicates -140 to $-160 \mathrm{dBm}$ signal strength along with multipath fading (assuming 1 $\mathrm{dB}$ noise figure). The red star is the spot where antenna was located during the experiment (static). The experiment was conducted starting indoors and lasted for about 30 minutes. No initialization in the outdoor or open sky was performed/required. The base station receiver was used only to provide the ephemeris data, similar to an assisted-GNSS situation. From Figure 1, one can see the rover receiver is mostly surrounded by concrete walls, but there are few windows in the ceiling. To the southwest (towards the right in Figure 1), there are some large glass windows near the entrance. Some pedestrian may walk around the data collection spot. This is considered as a typical indoor environment similar to big shopping malls.

The sky-plot of all the available satellites are shown in Figure 2. One can see the BDS GEO satellites are mostly from the south, which would result in stronger $\mathrm{C} / \mathrm{N}_{0}$ because of the windows. Regarding the GPS constellation, only PRN 26 has high elevation angle while all the others would be significantly attenuated or affected by the building or multipath.

\section{Data Processing and Result Analysis}

The left plot in Figure 3 is the $\mathrm{C} / \mathrm{N}_{0}$ profile for all tracked satellites. It is apparent that the $\mathrm{C} / \mathrm{N}_{0}$ are significantly affected by attenuation and multipath. Even for high elevation satellites, the $\mathrm{C} / \mathrm{N}_{0}$ curves are still highly time-varying. The right plot of Figure 3 shows the correlator outputs for BDS PRN 8 satellite. The two horizontal axes are the time (epochs) and code phase offsets, the range of code phase is $[-1,1]$ chips approximately 


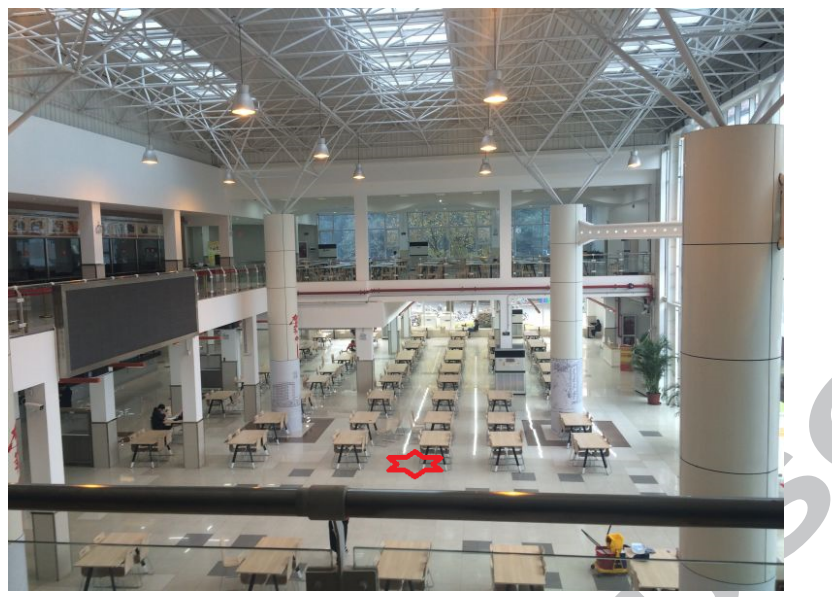

Figure 1: Picture of the indoor environment used for testing. The red star is the approximate location of the antenna. The picture is taken facing approximately southeast.

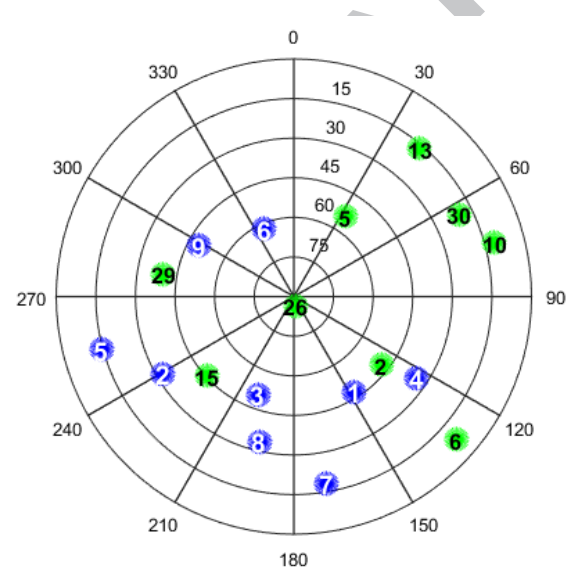

Figure 2: Skyplot of the constellations in-view. Blue marker indicates BDS constellation and green marker indicates GPS constellation.

centred around the peak of the correlation. It can be seen that the ideal correlation triangle is hardly noticeable. It follows that a traditional receiver that uses code phase measurements based on maximum likelihood estimation could produce a biased or deteriorated final solution.

The computed trajectories for the traditional and enhanced approaches are plotted in Google Maps in Figure 4. The ground truth is not surveyed due to the lack of professional equipment, and it is believed to have a accuracy 


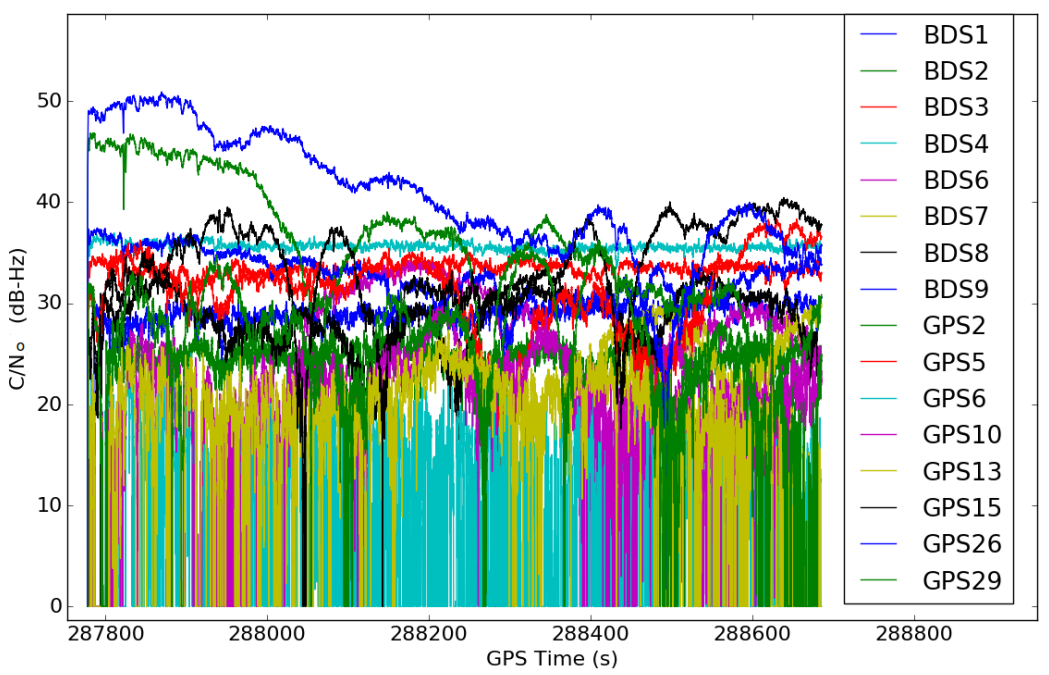

(a) $\mathrm{C} / \mathrm{N}_{0}$ for all GPS/BDS satellites

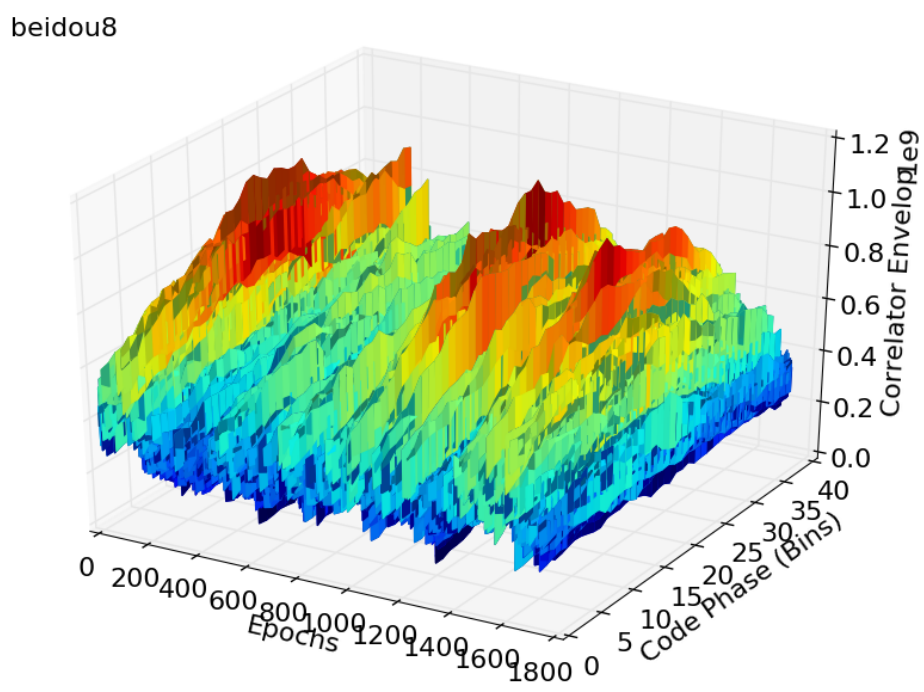

(b) BDS PRN-8 correlator outputs over time

Figure 3: $\mathrm{C} / \mathrm{N}_{0}$ values and the shape of correlator output 
better than $5 \mathrm{~m}$ which is considered enough for comparison purposes. For the first position fix, the traditional approach result is outside of the figure, but over time there is a tendency to converge towards the truth. Regarding the enhanced method, either the GPS-only or BDS-only solutions appears to be more robust than the traditional result. One can also find the first fix for the enhanced approach with different constellation are still around 20 to $30 \mathrm{~m}$ away from the truth. This is because the search step for the first fix is much coarse than the succeeding epochs. Detailed settings can be found in He \& Petovello (2014).

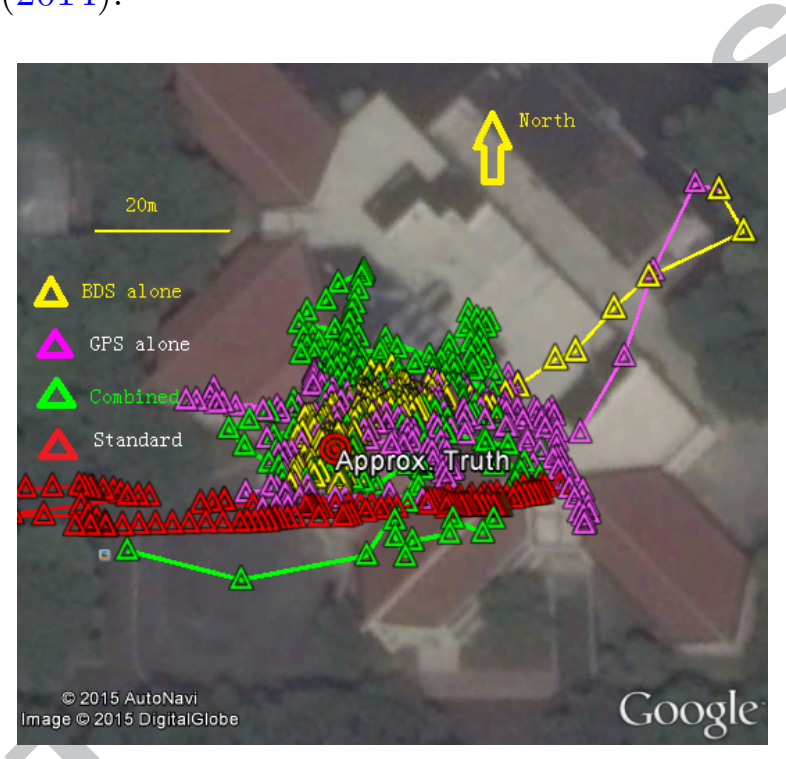

Figure 4: Trajectories for different approaches/constellations plotted in Google Maps. The red trace is from the standard approach using BDS+GPS. The remaining traces are obtained from the enhanced method: yellow for BDS alone, purple for GPS alone and green for BDS+GPS. The red concentric circle is the approximate true position.

Figure 5 shows the positioning errors for the traditional approach over time. One can easily observe that the initial fix has a very large bias especially in the vertical axis. Even though there is a tendency to converge in the horizontal direction, the vertical channel appear to continue drifting throughout the dataset. This is very likely due to the multipath effects. The biases in the multipath make the solution very prone to the biased position. Due to multipath the clock and height may not be able to be well estimated.

To the contrary, the enhanced approach uses all the correlator values, and estimates the solution based on all available information. The solution turns 

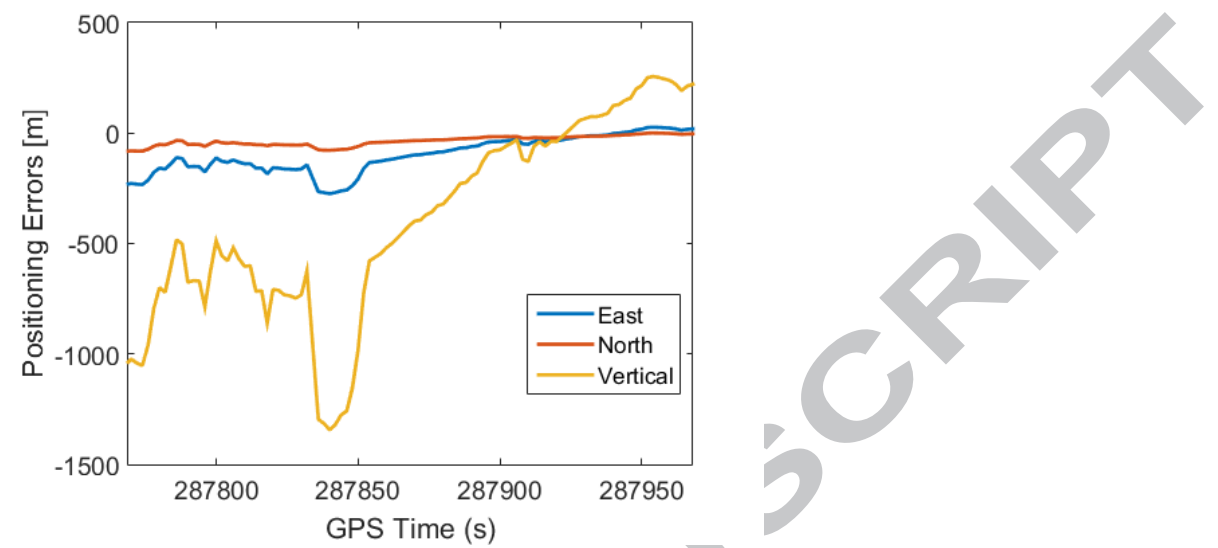

Figure 5: Positioning errors using tradition five state coarse time solution based on code phase measurements

out to be much better and more robust than the traditional receiver even using GPS or BDS constellation by themselves. The positioning errors are shown in Figure 6. From this figure, one can observe that the horizontal position are much better than vertical. The BDS-only solution has better horizontal position fixes than GPS in this indoor environment. The GEO satellites from the BDS constellation definitely help due to its relatively stronger signal power since they are likely less attenuated by the concrete walls. Regarding the vertical axis, the BDS alone solution has large vertical variations (about $80 \mathrm{~m}$ ), whereas the GPS alone solution has less variations (about $60 \mathrm{~m}$ maximum). When combining GPS/BDS, the observability in the vertical axis improves and the maximum variation is reduced to around $40 \mathrm{~m}$. Overall, the tendency of the solution to converge is more apparent when using both constellations. Detailed error statistics are summarized in Table 1 and Table 2.

Table 1 summarizes the mean positioning errors for all approaches. It is obvious that the traditional approach gives the worst result, especially for the vertical axis. Using enhanced approach, the horizontal solutions have significant improvement relative to the standard case regardless of what constellations are used with the mean error below $10 \mathrm{~m}$. The BDS alone solution has worse height estimation than GPS. The combined solution has the best performance in vertical axis, which is nearly unbiased.

Table 2 summarizes the standard deviation of all solutions. It can be seen that GPS has slightly bigger standard deviation than BDS, which is 

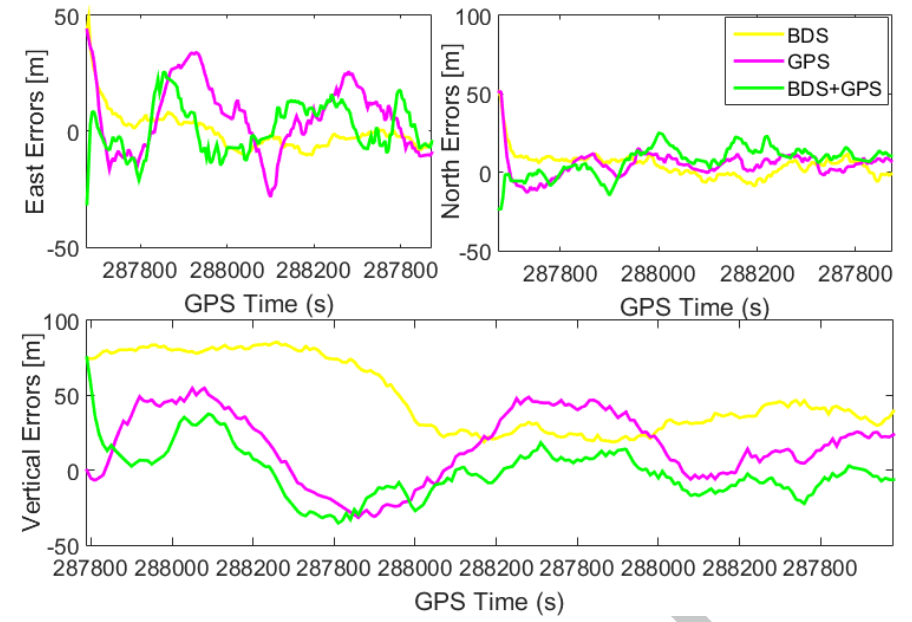

Figure 6: Positioning errors for different approaches/constellation; yellow indicates BDS alone solution, purple indicates GPS alone solution, green curve is the combined solution.

Table 1: Summary of mean positioning errors for all solutions

\begin{tabular}{|l|c|ccc|}
\hline \hline & Standard Rx & \multicolumn{4}{|c|}{ Enhanced Rx } \\
Axis & BDS+GPS & BDS & GPS & BDS+GPS \\
\hline East $(\mathrm{m})$ & 99.2 & 0.1 & 6.2 & 1.3 \\
North $(\mathrm{m})$ & -35.5 & 4.7 & 4.7 & 7.4 \\
Vertical $(\mathrm{m})$ & -395.0 & 49.1 & 16.3 & -1.3 \\
\hline \hline
\end{tabular}

reasonable since most GPS satellites will experience severe multipath and attenuation, whereas some BDS GEO satellites (south facing, and high elevation angle) will likely experience smaller effects. When combining both systems together, we can only see improvements in the vertical axis. The east and north direction has no apparently improvement, which is understandable since the performance of each constellation alone is already very good.

Finally, the coarse-time state estimates for all approaches are shown in Figure 7. From this figure, one can see that all the solutions have similar coarse-time estimates (within $0.01 \mathrm{~s}$, which translates to a maximum ranging error of around $8 \mathrm{~m}$ ). The enhanced method with GPS and BDS constellation has a faster convergence rate than using GPS or BDS constellations alone. The more reliable and accurate coarse-time is estimated, the better positioning results can be generated, as shown above. 
Table 2: Summary of standard deviation for all solutions

\begin{tabular}{|l|c|ccc|}
\hline \hline & Standard Rx & \multicolumn{3}{|c|}{ Enhanced Rx } \\
Axis & BDS+GPS & BDS & GPS & BDS+GPS \\
\hline East $(\mathrm{m})$ & 83.5 & 8.7 & 15.1 & 10.5 \\
North $(\mathrm{m})$ & 22.4 & 7.6 & 8.1 & 8.8 \\
Vertical $(\mathrm{m})$ & 436.7 & 24.0 & 23.5 & 17.3 \\
\hline \hline
\end{tabular}

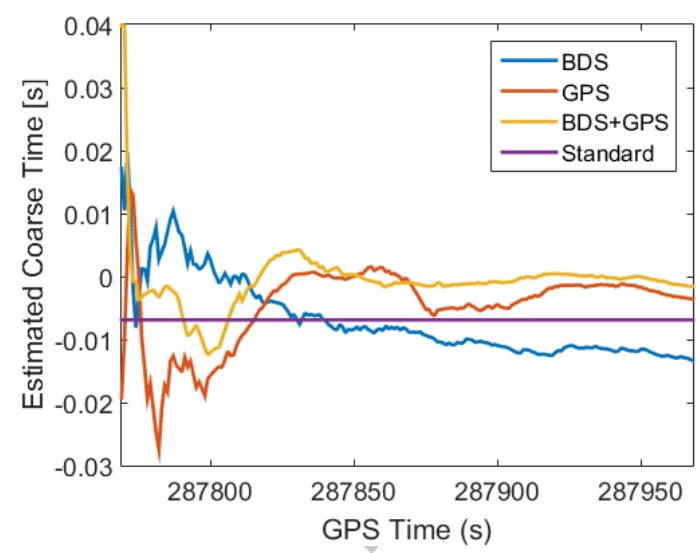

Figure 7: Estimated coarse time for different approaches/constellations

\section{Conclusions and Future Work}

In this paper, traditional and enhanced assisted-GPS/BDS algorithms have been discussed and evaluated with real indoor data collected in Shanghai, China. From the field test results, we observe that when non-line-of-sight signals dominate, the performance of traditional A-GPS/BDS receiver architectures does not provide satisfactory results. Based on the results shown above, the following conclusions can be made:

- Indoors, traditional receiver architectures that only use scalar code phase measurements cannot generate trust-worthy results, and position solutions are often largely biased. In contrast, the enhanced architecture gives much reliable and accurate position fixes, with improvements in height being especially significant.

- The horizontal positioning performance using BDS alone is slightly better than using GPS alone for the test scenarios considered herein. 
- The vertical axis has poorer performance when using BDS compared to the case using GPS alone. The largest deviation for BDS can be up to $80 \mathrm{~m}$, while $60 \mathrm{~m}$ for the GPS constellation.

- For the indoor scenarios considered herein (a shopping mall like environment), the enhanced assisted receiver can provide mean positioning errors less than 10 metres for all three axis when combining GPS and BDS. The convergence of the solutions is also more rapid.

- Enhanced assisted GPS/BDS receiver also shows better coarse-time estimation in indoors.

In the future, more environments will be tested and other constellations such as Glonass and Galileo will also be added.

\section{Acknowledgement}

The work is partially supported by the National Natural Science Foundation of China under Grant 61573242, in partly by Shanghai Science and Technology Committee under Grant 15511105100 and partly sponsored by Shanghai Pujiang Program (No. 14PJ1405000).

\section{Bibliography}

\section{References}

Axelrad, Penina, Bradley, Ben K, Donna, James, Mitchell, Megan, \& Mohiuddin, Shan. 2011. Collective detection and direct positioning using multiple GNSS satellites. Navigation, 58(4), 305-321.

Cheong, Joon Wayn, Wu, Jinghui, Dempster, Andrew G, \& Rizos, Chris. 2012. Assisted-GPS-based Snap-shot GPS Receiver with FFT-accelerated Collective Detection: Time Synchronisation and Search Space Analysis. Pages 2357-2370 of: 25th International Technical Meeting of the satellite Division of the Institute of Navigation (ION GNSS 2012), Nashville USA.

Closas, Pau, Fernández-Prades, Carles, Fernández-Rubio, Juan, et al. . 2007. Maximum likelihood estimation of position in GNSS. Signal Processing Letters, IEEE, 14(5), 359-362. 
De Wilde, Wim, Sleewaegen, Jean-Marie, Simsky, Andrew, Vandewiele, Christophe, Peeters, Edda, Grauwen, Jan, \& Boon, Frank. 2006. New fast signal acquisition unit for GPS/Galileo receivers. Pages 7-10 of: Proceedings of European Navigation Conference, ENC GNSS.

Gaggero, Pascal O, \& Borio, Daniele. 2008. Ultra-stable oscillators: limits of GNSS coherent integration. Pages 565-575 of: Proceedings of the 21st International Technical Meeting of the Satellite Division of The Institute of Navigation (ION GNSS 2008), vol. 8. Institute of Navigation.

He, Zhe. 2013. Receiver Architectures: What is a maximum likelihood vector tracking receiver? Inside GNSS, 8(4), 27-30.

He, Zhe, \& Petovello, Mark. 2014. Joint Detection and Estimation of Weak GNSS Signals with Application to Coarse Time Navigation. Pages 1554-1567 of: Proceedings of the 27th International Technical Meeting of The Satellite Division of the Institute of Navigation (ION GNSS+ 2014), Tampa USA.

He, Zhe, Petovello, MG, \& Lachapelle, Gérard. 2012. Improved Velocity Estimation with High-Sensitivity Receivers in Indoor Environments. Pages 1004-1016 of: Proceedings of the 25th International Technical Meeting of The Satellite Division of the Institute of Navigation (ION GNSS 2012). Institute of Navigation.

He, Zhe, Renaudin, Valérie, Petovello, Mark G, \& Lachapelle, Gérard. 2013. Use of High Sensitivity GNSS Receiver Doppler Measurements for Indoor Pedestrian Dead Reckoning. Sensors, 13(4), 4303-4326.

Hurd, WJ, Statman, JI, \& Vilnrotter, VA. 1987. High dynamic GPS receiver using maximum likelihood estimationand frequency tracking. Aerospace and Electronic Systems, IEEE Transactions on, 425-437.

Jin, Shuanggen, \& Najibi, Nasser. 2014. Surface scattering characteristics and snow accumulating-melting behaviors from GNSS reflectometry. Pages 25-28 of: Proc. Progress Electromagn. Res. Symp.(PIERS).

Lashley, Matthew, Bevly, David M, \& Hung, John Y. 2009. Performance analysis of vector tracking algorithms for weak GPS signals in high dynamics. IEEE Journal of Selected Topics in Signal Processing, 3(4), 661-673. 
P. Esteves, M. Saahmoudi, \& Ries, L. 2014. Collective Detection of MultiGNSS Signals:Vector-Acquisition Promises Sensitivity and Reliability Improvement. Inside GNSS, 9(3), 54-65.

Pany, Thomas, \& Eissfeller, Bernd. 2006. Use of a vector delay lock loop receiver for GNSS signal power analysis in bad signal conditions. Proceedings of IEEE/ION PLANS, 893-903.

Pany, Thomas, Riedl, B, Winkel, J, Wörz, T, Schweikert, R, Niedermeier, H, Lagrasta, S, López-Risueño, G, \& Jiménez-Baños, D. 2009. Coherent integration time: the longer, the better. Inside $G N S S, 4(6), 52-61$.

Parkinson, Bradford W. 1996. Progress in Astronautics and Aeronautics: Global Positioning System: Theory and Applications. Vol. 1. Washington DC: AIAA.

Proakis, J.G. 2001. Digital Communications. McGraw-Hill Series in Electrical and Computer Engineering. Computer Engineering. New York: McGraw-Hill.

Van Diggelen, Frank Stephen Tromp. 2009. A-GPS: Assisted GPS, GNSS, and $S B A S$. Artech House.

van Graas, Frank, Soloviev, Andrey, de Haag, Maarten Uijt, Gunawardena, Sandjeev, \& Braasch, Michael. 2005. Comparison of two approaches for GNSS receiver algorithms: batch processing and sequential processing considerations. Pages 200-211 of: Proceedings of 18th International Technical Meeting of the Satellite Division of the Institute of Navigation (IONGNSS) 2005, Long Beach, CA, September 13-16.

Zhuang, Yuan, Lan, Haiyu, Li, You, \& El-Sheimy, Naser. 2015. PDR/INS/WiFi integration based on handheld devices for indoor pedestrian navigation. Micromachines, 6(6), 793-812. 